\title{
Riesgos de la inflación semántica del bullying en los medios de comunicación y en el ámbito educativo
}

\section{The risks of the semantic inflation of bullying in the media and in the field of education}

DOI: https://doi.org/10.32870/dse.v0i24.1051

\section{Pablo Nahuel di Napoli* \\ Virginia Saez**}

\begin{abstract}
Resumen
Este trabajo analiza críticamente las prácticas discursivas sobre la problemática del bullying producidas en investigaciones académicas, en la prensa escrita y en la comunidad educativa. El corpus empírico está compuesto por 3,581 notas periodísticas de cuatro diarios de la Ciudad de La Plata y por 60 entrevistas semiestructuradas y nueve grupos focales realizados con estudiantes de escuelas secundarias en la provincia de Buenos Aires, Argentina. En primer lugar, se delimita brevemente el bullying como objeto de estudio y como perspectiva de análisis. Luego, se identifica y caracteriza el uso de dicho término que la prensa hace en sus coberturas mediáticas. Por último, se analizan los sentidos que las y los estudiantes construyen sobre la problemática en cuestión. Desde una perspectiva socioeducativa, por un lado, se identificó una convergencia discursiva que despliega una mirada individualizante y estigmatizante sobre las violencias en las escuelas; por otro lado, se observa una inflación semántica del término bullying para nominar situaciones diversas que exceden al fenómeno en cuestión. Se concluye que es necesario ser cauto en el uso de este vocablo y de ciertas premisas de su perspectiva que pueden contribuir, performativamente, a forjar aquello que postulan analíticamente.
\end{abstract}

Palabras clave: violencia - escuela secundaria - prensa - jóvenes estudiantes.

\section{Abstract}

This paper analyzes critically the discursive practices produced by academic research, the written press and the educational community on the phenomenon of bullying. The empirical corpus is comprised of 3581 journalistic notes from 4 newspapers of the City of La Plata, 60 semi-structured interviews and 9 focus groups with students from secondary schools in the province of Buenos Aires, Argentina. We defined

* Doctor en Ciencias Sociales. Líneas de investigación: conflictividad, convivencia y violencia en las escuelas; jóvenes y escuela secundaria, procesos de estigmatización social. Investigador Asistente del Consejo Nacional de Investigaciones Científicas y Técnicas (CONICET) COn sede de trabajo en el Instituto de Investigaciones en Ciencias de la Educación. Universidad de Buenos Aires. Argentina. pablodinapoli@filo.uba.ar

** Doctora en Ciencias de la Educación. Líneas de investigación: las relaciones entre los medios de comunicación y la escuela: la educación mediática en el sistema educativo, las representaciones mediáticas de la escuela y los efectos simbólicos que producen. Investigadora Asistente del Consejo Nacional de Investigaciones Científicas y Técnicas (CONICET) con sede de trabajo en el Instituto de Investigaciones en Ciencias de la Educación. Universidad de Buenos Aires. Argentina.virginiasaez27@gmail.com 
bullying briefly as an object of study and an approach to analysis, identified how the written press uses the term in its media coverage, and studied the meanings that students construct on the phenomenon in question. From a socio-educational perspective, we identified on the one hand a discursive convergence that deploys an individualizing and stigmatizing view of violence in schools, and on the other hand we observed a semantic inflation of the term bullying to name different situations that exceed the phenomenon in question. We conclude that is necessary to be cautious in using this word, and that certain assumptions from this perspective may contribute to shape, performatively, what they postulate analytically.

Keywords: violence - high school - mass media - young students.

\section{Introducción}

En América Latina se encuentra cada vez más extendido el término bullying ${ }^{1}$ para referirse de forma genérica a diversas situaciones de violencia que acontecen en el espacio escolar. Su uso ha tenido una amplia expansión en los medios de comunicación y en ciertos ámbitos profesionales especializados. Sin embargo, la problemática denominada buling conlleva una delimitación específica del fenómeno de las violencias en las escuelas, así como también encarna un enfoque particular de análisis.

Sostenemos que es necesario ser cautos en el uso de este vocablo y de ciertas premisas de su perspectiva que pueden contribuir, performativamente, a forjar aquello que postulan analíticamente. Las palabras expresan sentidos diversos en función de los usos y contextos en los cuales son pronunciadas. El simple acto cotidiano de nominar algo o a alguien contribuye a darle entidad y definirlo como tal en cuanto mayor reconocimiento tiene quien realiza la nominación (Bourdieu, 2014). En este artículo nos proponemos analizar qué se entiende por buling en el campo académico y cuáles son los usos y sentidos que los medios de comunicación y los actores de la comunidad educativa construyen a su alrededor. En primer lugar, haremos un recorrido breve por las investigaciones que indagan el buling, dando cuenta de su delimitación como objeto de estudio y los enfoques teórico-epistemológicos desde los cuales lo abordan. En segundo lugar, nos detendremos en el uso que los medios de comunicación gráficos hacen del término en sus coberturas mediáticas sobre situaciones de violencia en las escuelas. En tercer lugar, analizaremos los sentidos que los actores de la comunidad educativa, en particular, las y los estudiantes de educación secundaria, construyen sobre el fenómeno en cuestión. Por último, interpretaremos el entrelazamiento de sentidos entre el ámbito académico, el campo mediático y la percepción de los sujetos que transitan nuestras instituciones educativas.

El material empírico que presentamos es parte de tres investigaciones cualitativas ${ }^{2}$ realizadas en el marco del programa de investigación "Transformaciones Sociales, Subjetividad y

1 En adelante buling, para naturalizar el concepto en idioma español.

2 Se trata de las tesis doctorales de ambos autores y de la investigación posdoctoral del Dr. Pablo di Napoli. Las tres investigaciones fueron posibles gracias al financiamiento de becas de posgrado del Consejo Nacional Investigaciones Científicas y Técnicas (CONICET) de Argentina. 
Procesos Educativos" con sede en el Instituto de Investigaciones de Ciencias de la Educación de la Universidad de Buenos Aires, dirigido por la Dra. Carina V. Kaplan. El corpus de fuentes secundarias está conformado por 3,581 notas periodísticas de cuatro diarios (El Día, Hoy, Diagonales y Extra) de la Ciudad de La Plata, capital de la provincia de Buenos Aires, publicadas durante el periodo 1993-2011. El tratamiento de los datos se realizó en el marco del análisis socioeducativo del discurso (Martín, 2014).

El corpus de fuentes primarias está compuesto por 60 entrevistas semiestructuradas y nueve grupos focales realizados con estudiantes de cuatro escuelas secundarias de gestión estatal, entre 2012 y 2016. Dos de las instituciones están ubicadas en la localidad de Avellaneda y las otras dos en La Plata, provincia de Buenos Aires. El análisis de las entrevistas se llevó a cabo siguiendo los lineamientos del análisis temático, el cual supone el reconocimiento de patrones dentro de los datos donde los temas emergentes, en nuestro caso referido al buling, devienen en categorías analíticas (Fereday, Muir-Cochrane, 2006).

\section{El buling como objeto de estudio y como perspectiva de análisis}

Las primeras investigaciones sobre buling surgieron en los países escandinavos, a principios de la década de 1970, como consecuencia del suicidio de alumnos que habían declarado ser objeto de maltrato por parte de sus compañeros. El término original utilizado en dichos estudios fue mobbing, palabra que proviene del campo de la etología, y hace alusión a un ataque colectivo realizado por un grupo de animales contra otro de una especie distinta a la suya, que suele ser más grande que ellos (Olweus, 1996).

A partir de la proliferación de estos estudios en Gran Bretaña durante la década de 1980, aquel término fue remplazado por el de bullying (Smith, Sharp, 1994). En los años noventa, este tipo de investigaciones se expandió por Estados Unidos y varios países de Europa, siendo España uno de los referentes de habla hispana (Cerezo, 2009; Ortega, Mora-Merchán, 1997; Trianes, 2000). Si bien en Latinoamérica su abordaje es más reciente, en la última década se han realizado un gran número de pesquisas en diferentes países de la región (Román, Murillo, 2011; Herrera-López, Romera, Ortega-Ruiz, 2018). En el idioma español no existe una traducción literal del concepto y en distintas ocasiones es expresado a través de términos como "hostigamiento", "intimidación", "abuso" o "acoso" entre pares.

Esta breve referencia sobre la génesis del concepto resulta pertinente para rastrear las raíces desde las cuales se edifica la perspectiva del buling y se delimita su objeto de estudio. Inspirado inicialmente por la etología, este enfoque se nutre fundamentalmente de categorías del campo de la psicología experimental, próxima a la biología, que estudia cuestiones del comportamiento de los individuos.

Dan Olweus (1996), uno de los investigadores pioneros en este campo, sostiene que "un estudiante es objeto de hostigamiento cuando se halla expuesto(a), repetidamente y a lo largo 
del tiempo, a acciones negativas por parte de uno o más estudiantes" (1996: 360). Según este autor, una conducta negativa consiste en la intención explícita de infligir daño o molestia a otro, ya sea física, verbal o psíquicamente. Así, el fenómeno del buling se define a partir de tres características: a) la intención de una o varias personas de dañar a otra; b) la reiteración de este comportamiento a lo largo del tiempo, y c) la existencia de un desequilibrio de poder en la relación interpersonal entre la víctima y el hostigador. Del Rey y Ortega (2007) agregan una cuarta característica a la que denominan "ley del silencio", es decir que "las personas implicadas, directa o indirectamente, tienden a mantener en silencio lo que está sucediendo, lo que dificulta que deje de ocurrir" (2007: 78). Por su parte, Smith y Sharp (1994) sintetizan estas situaciones como abuso sistemático de poder.

Desde esta perspectiva, no se encuadran dentro de este concepto las agresiones que no necesariamente se producen de modo sistemático entre los mismos estudiantes o cuya relación de fuerzas se puede considerar simétrica. Bajo estas premisas, quedan por fuera del eje de indagación un cúmulo de situaciones de violencia que también acontecen en las escuelas (di Napoli, 2018).

Desde sus primeras indagaciones, Olweus (1998) ha diseñado un cuestionario anónimo autoadministrado (The Revised Olweus Bully/Victim Questionnaire) sobre hostigamiento y victimización, que fue adaptado y reproducido en varios estudios realizados en diferentes países (Menesini, 2009; Ortega, Mora-Merchán, 1997; Resett, 2011; Smith, Sharp, 1994; Zequinão, Medeiros, Pereira, Cardoso, 2016). De sus resultados se pueden plantear una serie de consideraciones generales. Entre las formas de maltrato más comunes se mencionan, en primer lugar, los insultos, apodos y sobrenombres; luego los golpes, agresiones físicas y robos, y en tercer lugar, las amenazas, rumores y el aislamiento social. Estos estudios concluyen que el número de víctimas de hostigamiento disminuye a medida que se asciende en los cursos escolares, es decir que el buling está más presente en los primeros años de escolaridad.

La dinámica del buling se focaliza en un juego de tres roles. Por un lado, la figura de la víctima, que es objeto del hostigamiento y, por el otro, el victimario, que es quien obra las conductas de intimidación. Existe un tercer papel que representa a aquellos que son testigos o espectadores de los hostigamientos, pero que no participan directamente en ellos (Del Rey, Ortega, 2007; Olweus, 1996). Sin embargo, la distinción entre víctima y victimario no siempre resulta clara. Zequinão et al. (2016) mencionan que existe una combinación entre autor y víctima relativamente alta, constatándose una ambigüedad que no sugiere una clara diferenciación entre estos roles. Olweus (1996) y Ortega Ruiz (2006) refieren a la existencia de "víctimas proactiva" o de "agresores victimizados", es decir, de estudiantes que participan de ambas posiciones en distintas ocasiones.

Las investigaciones sobre buling abordan las relaciones interpersonales predominantemente desde aspectos psicológicos y de la personalidad de los individuos involucrados, sin en- 
marcar dichas relaciones en un contexto más amplio de interdependencias. Gran parte de las investigaciones (Castillo, Pacheco, 2008; Castro, 2013; Cerezo, 2009; Defensor del Pueblo, 2007; Farrington, Lösel, Ttofi, Theodorakis, 2012; Menesini, 2009; Olweus, 1998) brindan, con pequeñas variaciones, una caracterización del perfil psíquico típico de las víctimas y de los agresores. Allí, se describe generalmente a las víctimas como personas inseguras, ansiosas y con un patrón sumiso; mientras que los hostigadores muestran una pauta agresiva basada en la necesidad de poder y dominio, la satisfacción de causar daño y el objetivo de apoderarse de objetos de la víctima.

La aplicación del cuestionario creado por Olweus (1996) en diferentes partes del mundo ha permitido consolidar dicho instrumento de recolección de datos haciendo posible comparaciones entre distintos países e, incluso, realizar los pocos estudios longitudinales que existen sobre el tema de violencia en las escuelas. Sin embargo, como sostiene Paulín (2015), una de las dificultades de estas pesquisas radica en la validez de la definición operacional de lo que se considera violencia.

\section{El uso del término buling en los medios de comunicación}

A partir del estudio sociohistórico sobre la cobertura de las violencias en las escuelas en la prensa escrita de la ciudad de La Plata durante el periodo de 1993-2011 (Saez, 2019), se identificó que la representación de los episodios violentos entre estudiantes homologa situaciones diversas (peleas, intimidaciones, matanzas). Es recién a partir del año 2008 que se empieza a nominar, de forma reiterada, las situaciones de violencia entre estudiantes como buling. Dicho año, coincide con la fecha en la cual Herrera-López, Romera y Ortega-Ruiz (2018) observan un salto cuantitativo en la publicación de investigaciones sobre buling en América Latina (pasa de una publicación en 2007 a diez en 2008) que continúa creciendo al menos hasta 2015 inclusive (con 47 artículos encontrados).

Ahora bien, si nos detenemos en cada uno de los casos referenciados en las notas periodísticas, advertimos que no todos se enmarcan en las definiciones que se proponen desde el campo académico al que hicimos referencia en el apartado anterior. Esbozamos como hipótesis interpretativa que se produce una inflación de este término. Asimismo, esta amplificación del término se corresponde con el desarrollo de una veta comercial, ya sea en el mercado editorial con libros que, a modo de decálogo, determinan si "su hijo es matón o víctima"; o en nuevas unidades de negocio de empresas, ONG o fundaciones que ofrecen, por ejemplo, seguros contra buling para las escuelas ${ }^{3}$ o talleres de capacitación para docentes.

Durante todo el periodo analizado, se observa la convergencia de un discurso que reduce la problemática de la violencia a acciones individuales, sin tomar en consideración las tramas

3 Clarín, 13 de octubre de 2014. "Venden seguros contra el bullying a los colegios". http://www.clarin.com/sociedad/Venden-seguros-bullyingcolegios_0_1229277361.html 
institucionales y socioculturales más amplias (Kaplan, 2008b). Dicha forma de presentación se refuerza con la representación de los vínculos entre los estudiantes en términos de víctimas y victimarios.

\section{El buling como un suceso novedoso}

Con este término se visibiliza una forma inédita de violencia entre pares que está en franco crecimiento en el país, veamos algunas menciones.

El fenómeno del bullying, la nueva violencia entre chicos (El Día, 30 de enero de 2008).

El "bullying" es, según estos datos, un fenómeno en alza, ya que hace una década la cifra de agredidos no era de más de uno o dos de cada diez (El Día, 6 de octubre de 2004).

Esta modalidad de violencia se presenta de forma espectacularizada y asociada a un discurso emotivo. Estos modos comunicativos son frecuentes para la visibilización de los hechos sociales (Saez, 2020). En el caso de buling se presenta de la siguiente manera:

MIEDO EN LAS AULAS (EI Día, 24 de noviembre de 2011).

La habitación del pánico (EI Día, 5 de marzo de 2009).

Fruto de la sociedad en que vivimos, la violencia escolar se ha convertido en una de las tantas pesadillas con las que los habitantes de las grandes ciudades deben lidiarla prácticamente a diario. Las peleas entre compañeros de curso, los insultos y hasta el hostigamiento entre pares -bullying, que le dicensorprenden (Hoy, 22 de mayo de 2009).

\section{Psicopatologización de la vida escolar}

El buling como perspectiva que circula en el discurso mediático tiene efectos simbólicos sobre la representación de los actores que habitan el campo educativo. Los modos de designar esta forma de violencia entre pares refieren al campo de la salud y lo destacan como una enfermedad social, una epidemia de difícil solución cuyos síntomas pueden identificarse psicopáticamente en los estudiantes. Veamos algunas notas:

Acoso escolar: más frecuente al inicio del secundario. Es un flagelo más común entre los alumnos varones (El Día, 5 de abril de 2011).

UNA EPIDEMIA SILENCIOSA (Hoy, 10 de mayo de 2009).

LOS SÍNTOMAS. ¿A qué síntomas hay que estar atentos para prevenir este tipo de hostigamiento? Según Vázquez, el insomnio, los cambios de humor repentinos, el llanto inmotivado, la tristeza, la depresión o el descenso del rendimiento escolar son algunas de las señales de alarma a las que conviene estar atentos (EI Día, 5 de abril de 2011). 
Ahora bien, la aparición del buling en la prensa se dio desde el discurso autorizado del campo científico, esto tiene correlato con nuestra hipótesis interpretativa acerca de la inflación y la veta comercial del término. En el corpus de notas analizadas aparece con recurrencia la mención a fuentes de legitimación de la información provenientes del exterior. Se mencionan variados estudios y organismos, como el Programa para la Evaluación Internacional de Alumnos (PISA) (EI Día, 10 de junio de 2011); cita de profesionales de la UAM (El Día, 2 de noviembre de 2011); un estudio de la Organización de las Naciones Unidas para la Educación, la Ciencia y la Cultura, UNESCO (EI Día, 2 de noviembre de 2011); y una investigación divulgada por UNICEF. Resulta relevante destacar este aspecto dado que, como sostiene Bourdieu (2006), es parte de estrategias en la apuesta por el monopolio de la autoridad científica, es decir, la capacidad técnica y el poder social de hablar y de actuar legítimamente. Estas fuentes operan legitimando la narrativa de que hay más violencia en las aulas y que es necesario modificar esta situación.

Surge la inquietud sobre ¿qué prácticas se están dando por supuestas al legitimar las prácticas discursivas utilizando como fuentes de información estudios y organismos internacionales? La construcción de la violencia en el espacio escolar desde informes y estudios de organismos exteriores sugiere pensar en cómo, desde la mirada internacional, están proponiendo ciertos campos de acción y estructuran el orden de las cosas y el campo de lo posible en las experiencias de violencia en el espacio escolar. Es necesario resaltar la necesidad de ser cuidadosos y tomar muchos recaudos cuando se traslada mecánicamente un enfoque y una terminología a nuestros contextos. En este acto de dar voz a los estudios que provienen del exterior y desestimar o no presentar los que se realizan a nivel nacional y las voces de profesionales de las escuelas, se está produciendo una mirada sobre la violencia que es homologable a otras sociedades, porque se habla de igual forma de la sociedad argentina que de otras. Por otro lado, se da de manera tácita una autorización a los estudios exteriores más que a los nacionales. En este sentido, es necesario recuperar la relación individuo-sociedad que manifiestan y dan por supuesta estas prácticas discursivas. Estas narrativas parecen suponer una homologación de las configuraciones sociales (Elias, 1999) en las que se desenvuelven los individuos, dado que los análisis sobre la violencia en la escuela pueden ser trasladables.

Ahora bien, los expertos que aparecen son exclusivamente de la rama biomédica o psicológica: médico pediatra (El Día, 3 de julio de 2011), médico especialista en psiquiatría infantil (EI Día, 3 de julio de 2011), psicóloga especializada en adolescencia y familia (El Día, 2 de noviembre de 2011), Equipo Bullying Cero Argentina (El Día, 24 de noviembre de 2011), psicólogo especialista en trastornos de conductas de la niñez y la adolescencia (El Día, 9 de mayo de 2011) y un experto en violencia escolar (EI Día, 24 de noviembre de 2011) del cual no se especifica profesión. Esto se corresponde con las disciplinas que encabezan las investigaciones sobre buling que vimos en el apartado anterior, fundamentalmente psicología y medicina interconectadas en el área de salud mental. 
Nos preguntamos entonces ¿qué efectos tiene la apelación de los periodistas al discurso científico para argumentar sus posicionamientos en el discurso mediático sobre el buling?

La utilización "neutral" de diversos estudios, sin la crítica teórica de los "datos" preconcebidos o sin vincularlos con la construcción del objeto de conocimiento, ha dado lugar a indagaciones sobre este fenómeno que solamente sistematizan el sentido común. Así, desde las prácticas discursivas de la prensa platense se presentan voces científicas como discursos autorizados para describir y prescribir el buling.

\section{La visión mediática sobre la operatoria del buling}

Los discursos de los medios describen una "metodología" sobre cómo este fenómeno se lleva a cabo. En coincidencia con los estudios descritos en apartados anteriores, las coberturas tipifican los hechos como conductas repetitivas que tienen que ver con la intimidación, la tiranización, el aislamiento, la amenaza, los insultos sobre una víctima o víctimas ya marcadas. Veamos cómo lo hacen:

El "bullying" comienza por manifestarse de una manera sutil, mediante bromas y novatadas, para ir haciéndose cada vez más cruel (burlas, patadas, empujones). Una vez que el agresor controla a la víctima, controla también al grupo (El Día, 6 de octubre de 2004).

A diferencia de un conflicto ocasional, la agresión en el marco del acoso escolar viene acompañada de una expectativa de que ese tipo de ataque se va a repetir (EI Día, 5 de abril de 2011).

Esta forma de describir el fenómeno alude a una dinámica con roles fijos: víctima u hostigado, victimario u hostigador, espectadores y seguidores, en donde se mencionan en mayor parte varones. Este aspecto es coincidente con las investigaciones sobre el tema. Observemos algunas evidencias:

Hay un momento crítico en materia de acoso escolar: los primeros años del secundario. Así lo indican especialistas, que agregan que se trata de un flagelo que con más frecuencia es protagonizado -tanto en el rol de víctimas como de victimarios- por alumnos varones. Y que afecta a cursos enteros, ya que las situaciones asociadas a este problema dejan huella tanto en sus protagonistas como en los testigos (El Día, 5 de abril de 2011).

Mientras ayer resonaban los ecos del caso de las chicas que se pelearon en Facebook y una terminó apuñalada, varios especialistas consultados por este medio admitían que la violencia entre los más jóvenes es una problemática que crece y que suele ubicar a los adolescentes tanto en los lugares de víctimas como en los de victimarios (El Día, 24 de noviembre de 2011).

Para su visibilización se presentan infografías que abonan a esta mirada. A continuación presentamos un ejemplo: 
Infografía 1. El círculo del bullying

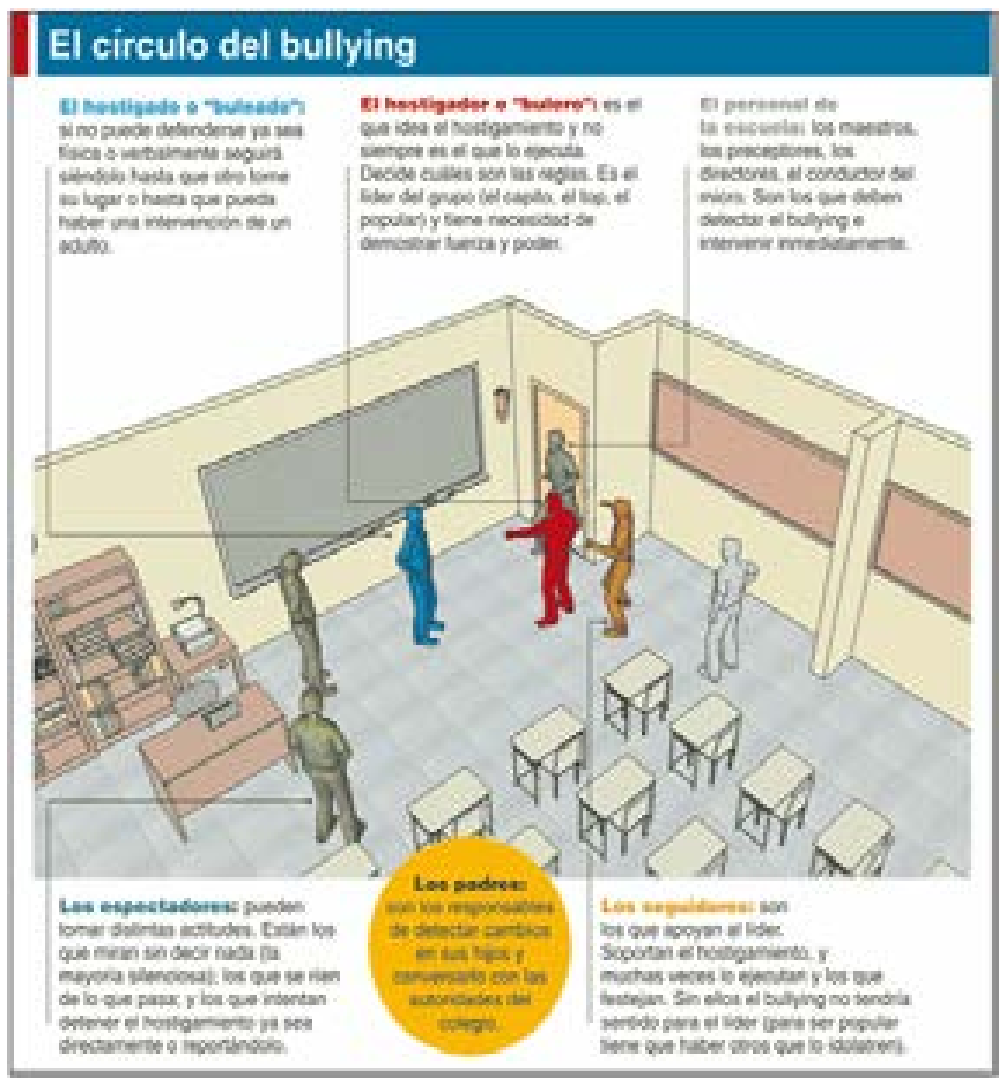

Fuente: El Día, 24-11-2011.

En su caracterización se hace mención a las motivaciones del agresor - "tiene necesidad de mostrar fuerza y poder" (EI Día, 24 de noviembre de 2011; El Día, 10 de septiembre de 2010)—y las secuelas que dejan en la víctima las situaciones de acoso.

Como se mencionó anteriormente, es una terminología que no ha sido construida en el campo educativo-pedagógico sino que es propia de otros campos, y tiene dificultad para dar respuesta a las problemáticas dentro del ámbito escolar. Desde esta perspectiva del buling, reforzando el par binario víctima-victimario, se intenta construir una tipología de las víctimas (alumnos inseguros, indefensos, entre otras características) y de los agresores (controladores, líderes de grupo, el capito, el top, el popular, con necesidad de demostrar fuerza y poder, etc.). Estas supuestas cualidades intrínsecas de un tipo de niño-niña-joven actúan como predictivas del comportamiento del sujeto en el mundo social.

\section{Los estudiantes y la operatoria del buling}

La visión mediática establece una clara asociación del buling con la disponibilidad de los jóvenes con la tecnología que muchas veces se describe como "adicción a los videojuegos" (El Día, 5 de marzo de 2009). En otras notas se menciona: 
Mientras las peleas escolares no cesan, en Internet se multiplican los sitios que promueven las disputas entre adolescentes (EI Día, 24 de noviembre de 2011).

Hace años que en la cultura televisiva se instaló eso de burlarse del otro subrayando su vulnerabilidad. De alguna forma lo de [Marcelo] Tinelli y otros ha ido habilitando la burla al interior del ámbito educativo (El Día, 10 de mayo de 2009).

Así pues, este fenómeno visibilizado como "buling" fue mutando por el de "ciberbuling" o "acoso virtual" con la expansión de estas prácticas en las redes sociales, en donde el límite simbólico del adentro y el afuera escolar se fue haciendo más difuso: "Aulas y redes sociales, dos ámbitos de agresión juvenil" (EI Día, 24 de noviembre de 2011).

Según los expertos citados por la prensa, las nuevas formas de comunicación que provee internet hacen visible una violencia que ya existía en el ámbito privado.

CIBERBULLYING. Según los especialistas, el bullying se ejerce cuando no están los adultos, y de forma repetida, generalmente en la escuela. Aunque con la expansión de las nuevas tecnologías como Internet o la telefonía móvil, también continúa fuera del ámbito educativo. "Antes el chico llegaba a la casa y estaba a salvo, ya que la víctima cree que nadie puede ayudarlo. Pero ahora el acoso sigue a través de Internet, del chat, de las redes sociales, de los mensajes de texto, es lo que llaman ciberbullying, que no es otra cosa que el mismo bullying", reparó Kelly (EI Día, 5 de abril de 2011).

Además de la creación de grupos en las redes sociales con la intención de agredir a terceros, otra metodología frecuente suele ser el ingreso a páginas personales para dejar insultos o burlas bajo el amparo de seudónimos. Algunas iniciativas incluyen la creación de Fotologs de terceros con esa misma finalidad. También en el portal de videos YouTube se puede ver con facilidad numerosos videos de peleas en aulas, en patios de escuela o en la calle (EI Día, 24 de noviembre de 2011).

Lo que dice la especialista sobre la violencia escolar también integra ese universo violento que salpica cada día a nuestra sociedad. Según varios estudios, el uso de Internet y de la tecnología para los jóvenes se ha visto distorsionado en este último tiempo por las malas herramientas de los teléfonos celulares, las redes sociales o los soportes de video en red, que han hecho que se cree una nueva forma de amedrentar y aterrorizar, convirtiendo cada día a miles de jóvenes en personas acosadas mediante las nuevas tecnologías. Varios estudios realizados en todo el mundo revelan que más de la tercera parte de la gente joven ha experimentado o ha sido víctima de ciberbullying (Hoy, 24 de octubre de 2010).

Contra delitos y acosos hoy por una Internet más segura. En cinco continentes hoy es el Día Internacional por una Internet Segura. La jornada advierte y previene contra el "ciberbullying" o acoso en las computadoras que los chicos usan en las escuelas. También en rechazo de contenidos racistas o de pornografía infantil en la Red (Diagonales, 6 de febrero de 2009).

Las consecuencias que se mencionan son variadas y van desde las emocionales y físicas hasta las fatales. Se destaca que la víctima puede tener alucinaciones, y se la describe como 
débil, psicótica, con "trastornos de autoencierro", "adicción a los videojuegos". Proyectan que pueden tener un brote psicótico o matar a sus compañeros.

La adicción a los videojuegos genera preocupación a nivel mundial. [...] Sus chicos han sufrido durante toda la primaria acoso escolar -bullying-, los ven muy débiles para enfrentar al mundo y los sobreprotegen". No les imponen límites por el temor a cómo puedan reaccionar. "Vos escuchás las noticias y llega un momento en que no sabés qué hacer -confiesa el padre de Juan-. Teníamos miedo de que si le sacábamos la computadora o la televisión se volcara a las drogas. Hablamos con mi señora y preferimos el que creímos el mal menor". Rotenberg agrega: "Siempre hay alguien en la familia en complicidad, que le acerca la comida y lo atiende. Los chicos terminan siendo pequeños (o grandes) tiranos en su casa, pero al obligarlos a salir pueden llegar a descompensarse, tener un brote psicótico y, si se los fuerza, hasta llegar a matar" (El Día, 5 de marzo de 2009).

Tengo un caso de una señora que adoptó a su hijo de bebé. Sentía que era una pertenencia y le transmitió desconfianza del mundo -comenta Rotenberg-. Ese chico dejó el colegio, no sale de la habitación y dice que si lo obligan, va a matar a todos sus compañeros. Atiendo a la madre y a la abuela, y estamos logrando que el chico estudie con profesores particulares en su casa (El Día, 5 de marzo de 2009).

Los chicos con trastorno de autoencierro sienten una gran desconfianza hacia el mundo, creen que cualquiera puede dañarlos. [...] Otros donde fui a la casa y tuve que enfrentarme a un chico amenazando a la madre con un cuchillo para que no me dejara entrar. El punto clave es la constancia: cuando ven que no desaparecés, te dejan entrar en su vida y en su familia (EI Día, 5 de marzo de 2009).

Este tipo de violencia tiene sobre todo consecuencias emocionales (51\% de los casos), pero también físicas (13\%). Los menores que sufren ciberbullying bajan además su rendimiento escolar (13\%), se aíslan socialmente (6\%) y faltan a clase por miedo (3\%) (Hoy, 22 de mayo de 2009).

Así lo afirman expertos en esta problemática, que volvió a instalarse en las últimas horas en el centro de la escena a partir de un caso de un chico de 12 años, de la localidad de Temperley, que se suicidó de un disparo en la cabeza. Desde algunos testimonios familiares se apuntó a la escuela y se afirmó que el chico (Víctor Felette) había padecido acoso escolar de parte de sus compañeros en clases de Educación Física a las que no quería asistir. Con todo, el caso tiene también aristas más complejas que actualmente se investigan. Y no está claro el rol que el presunto acoso escolar pudo jugar en él (El Día, 5 de abril de 2011). ESTRÉS Y DEPRESIÓN. “Uno de los cuadros más comunes que se desprenden del bullying es la depresión", explicó la médica pediatra Flavia Sinigagliesi, miembro del Centro de Investigaciones del Desarrollo Psiconeurológico (CIDEP) (Hoy, 22 de mayo de 2009).

Acá lo interesante también es cómo la prensa presenta casos extremos que terminan en muerte. 


\section{Las soluciones propuestas frente a las situaciones de buling}

En correlato con esta tipificación de cualidades caracterológicas de los acosadores, las soluciones propuestas desde el discurso mediático privilegian el cambio de escuela a la que asiste la "víctima". En consonancia con la mirada individualizante que prima, no aparecen propuestas que incluyan el trabajo con la comunidad educativa. Veamos algunos ejemplos:

Durante los primeros años de la secundaria sufrí las agresiones constantes de un grupo de compañeros con los que varias veces intenté llevarme bien, pero de los que jamás encontré respuestas más allá del hostigamiento. Al principio creí que toda esa pesadilla se podía revertir con el paso del tiempo, pero fue cada vez peor y tuve que cambiarme de escuela (El Día, 10 de mayo de 2009).

EL CAMBIO DE ESCUELA. "Si la escuela no toma conciencia de los riesgos de la violencia escolar, y no se puede avanzar en ese sentido, entonces la solución es cambiar de escuela al chico que está siendo acosado por sus compañeros, y asistirlo psicológicamente", explicó la profesora Mariana Kelly, del Equipo Bullying Cero (EI Día, 10 de septiembre de 2010).

"El caso de Matías — un chico que, tras ser acosado por sus compañeros durante toda la primaria, consiguió pedir ayuda y cambiarse de establecimiento educativo- logró conmover a los talleristas (El Día, 10 de septiembre de 2010).

Asimismo, esta forma de violencia en el discurso mediático connota un sesgo criminológico. En las nominaciones se define esta práctica como una conducta delictiva:

Gobiernos, empresas e instituciones de más de cincuenta y cinco países desarrollan hoy el Día Internacional por una Internet Segura para prevenir contra el 'ciberbullying' o acoso escolar en la red. También para sensibilizar a padres, educadores y menores sobre la existencia de riesgos en la Red y promover un uso responsable y seguro de las nuevas tecnologías y evitar las conductas delictivas como el acoso escolar (Diagonales, 2009).

Es importante detenerse a analizar estas afirmaciones prejuiciosas y criminalizantes. El buling es una categoría que bajo esta operación discursiva asocia jóvenes, acoso y delito, produciendo una judicialización de la vida social y educativa.

En los discursos de la prensa platense se describe el buling con una "aparente neutralidad ideológica y política de la categoría naturalizada de buling, a través de la cual se esencializa la cuestión social" (Kaplan, 2014: 2). Es presentado como fenómeno individualizante, sin situar la discusión en lo relacional, en las dimensiones históricas y en los contextos donde las biografías se anclan y encuentran su explicación y sentidos. Pensando con Carina Kaplan, podemos decir que hay una línea de continuidad histórica entre la tesis central del determinismo biológico (o del racismo biologicista) y el buling. En líneas generales, ambos enfoques 
coinciden en el hecho de aseverar que se nace o no se nace violento o bien, que se está o no predispuesto a la convivencia social.

\section{Las percepciones de los actores de la comunidad educativa}

La inflación, diversificación y abuso semántico del vocablo hace esquivo su significado también para los actores de la comunidad educativa. La imprecisión y "confusión" mediática se ve reflejada en las respuestas del estudiantado de las cuatro escuelas secundarias donde realizamos trabajo de campo. ${ }^{4}$ Era común que, cuando nos presentábamos en las instituciones, al contarles que trabajábamos sobre temas de conflictividad y violencias en las escuelas, los agentes educativos (directivos, docentes y preceptores) respondiera con frases como "claro, ustedes trabajan el tema del buling" o"ah, ustedes están estudiando el buling". En el caso de las y los estudiantes, cuando surgía el tema en las entrevistas, el cual no estaba pautado de antemano, les preguntamos qué significaba. Nos solían decir que no sabían "muy bien el significado de esa palabra", "porque te dicen muchas versiones".

P: ¿Qué significa bullying?

$\mathrm{H}$ : Es una persona violenta, que no le importa la otra persona, supuestamente es adicta al alcohol, a las drogas. Que maltrata sin pensar en los demás, piensa en él, tiene el ego muy alto, es popular. (Héctor, 5to. Año, Escuela 1 - Avellaneda).

C: Es como maltratar, humillar a otra persona, molestarla, pegarle. Eso todo el tiempo.

$P:$ ¿Y eso quién lo decía?

C: La directora, pero nosotros no lo vemos así. Es un pibe común que tiene sus problemas.

Capaz que viene y se descarga acá. Se descarga acá en el curso y bueno, estamos nosotros acá (Camilo, 5to. Año, Escuela 1- Avellaneda).

L: Me dicen muchas cosas. Bullying es el agresivo, el que le pega a la gente inferior o cosas así.

Es que nunca lo puedo llegar a tener muy claro porque te dicen muchas versiones.

$P:$ ¿Yvos lo ves así?

L: En cosas que hacemos, pero... Ya creo que... Bullying ya es tener una enfermedad.

(Luciano, 5to. Año, Escuela 1 - Avellaneda).

En los relatos de las y los estudiantes se evidencia cómo se plasma el discurso mediático y socialmente instalado sobre el buling que describimos en el apartado anterior. El problema es asociado, más que a entramados sociorelacionales, a comportamientos individuales o a características de la personalidad, calificando al sujeto como "violento", "descontrolado", "adicto", entre otras adjetivaciones. La última frase de Luciano es esclarecedora sobre la perspectiva desde la cual se entiende y percibe este fenómeno en el plano del sentido común. Ya no se trata de un tipo de vínculo interpersonal dañino, sino de una enfermedad que posee individualmente quien ejerce maltrato.

4 Al final de cada uno de los testimonios se señalará el nombre de los estudiantes (en el caso de las entrevistas individuales), el año de cursada, la escuela y la localidad en la cual se ubica). 
Al hablar sobre situaciones de violencia que ocurrían en la escuela, algunos estudiantes mencionaron casos que tipificaban como buling:

P: Vos decís que en el turno mañana hay bullying, ¿qué es bullying para vos?

E: Cuando maltratan a chicos que son diferentes, no necesariamente diferentes, son diferentes a uno mismo, y que le afecta por ejemplo a este chico que le decían que era gay le reafectó, yo lo vi llorando, y se tuvo que cambiar a otro colegio privado (Helena, 5to. Año, Escuela 2 Avellaneda)

E: ¿Qué otras situaciones de violencia me podrían mencionar?

A5: Yo conocí a una chica, de otro lado, que era compañera de mi prima, hace tres años, bueno, la chica sí, era gordita, todo, bien, era buenísima... es buenísima, pero le empezaron a decir un montón de cosas por Twitter, por Facebook, le hacían la vida imposible.

B3: Bullying le hacían.

E: ¿Era bullying eso?

A5: Sí, y todo el colegio se burlaba de ella, y bueno, la chica terminó siendo anoréxica y ahora está en rehabilitación, porque directamente se lo tomó mal y terminó... terminó haciendo ella... daño, se terminó haciendo daño a ella misma, siendo anoréxica, bulímica.

E: ¿Ustedes dicen que dejó la escuela?

A5: Sí, sí, dejó.

(Grupo focal, 6to. Año, Escuela 3 - La Plata).

En ambos casos, de extrema gravedad, se trata de estudiantes que fueron maltratados de forma reiterada por otro grupo de compañeros, ya sea dentro de la escuela o por redes sociales virtuales, causándoles profundos daños subjetivos que los llevaron a cambiar o dejar la escuela. Por un lado, podríamos decir que estos dos casos se enmarcan dentro de la definición clásica de buling que utilizan las investigaciones académicas. Por otro lado, el relato de los estudiantes deja en evidencia las dificultades que encuentran las instituciones educativas para abordar pedagógicamente estas situaciones, siendo una salida común el cambio o abandono de la escuela, tal como relataban los testimonios citados en la prensa gráfica.

Ahora bien, el término también es utilizado por los propios estudiantes para describir otro tipo de situaciones como la interacción entre docentes y alumnos o vínculos familiares que transcurren fuera de la escuela.

P: Recién me hablaban de bullying, ¿qué es para ustedes el bullying?

Joaquín: Yo creo que la palabra bullying hace referencia al acoso escolar pero también la usamos fuera del colegio, cuando hablamos de, no sé, un hermano molesta al otro, bueno, le hace bullying porque no se dice "lo acosó de alguna manera".

Luis: ¿Puedo salir al recreo?

P: Sí

Matías A.: Se va a ver a la novia, qué gato.

Cristian: Mi amor, vení rápido.

Joaquín: Además como que la usamos para todos los días: "uy, hiciste bullying" o "le hizo bullying a tal". 
(Grupo focal, 5to. Año, Escuela 4 - La Plata).

A2: Hay un profesor al que le hacen mucho... hacemos mucho bullying.

$P$ : ¿Le hacen mucho bullying?

B3: No le hacemos bullying...

A2: Es de Geografía.

B3: Jodemos, pero no es que bullying, no...

A2: Sí que le hacemos...

A3: O sea, al principio le hacíamos bullying, ahora como nos encariñamos un poquito más, nos da un poco más de pena.

B2: Sí, porque es re bueno.

$P:$ ¿Qué es el bullying para ustedes?

B1: Le tiraban papeles [superposición de voces].

B3: Bullying es pegar, molestar, decirle... todos a un misma persona.

A2: Y lo hacíamos con el profe. Lo seguimos haciendo a veces, pero con otro...

B3: Yo también...

A5: Yo creo que llega un momento que ya te da lástima...

B2: Da lástima.

(Grupo focal, 6to. Año, Escuela 2 - Avellaneda)

Los propios jóvenes reconocen que en la actualidad la palabra buling es utilizada por ellos para referirse a acciones y situaciones muy diversas. En el grupo focal de los estudiantes de 6 to. año de la escuela 2 se refleja una discusión entre los estudiantes sobre si su forma de comportarse frente al docente o "lo que le hacen" es buling. Por ejemplo, este último caso, desde el aspecto conceptual que desarrollamos en el primer apartado, no podría encuadrarse dentro de ese término ya que están refiriéndose a un vínculo que no es entre pares. Sin embargo, la imprecisión que opera en los discursos sociales hegemónicos genera que cualquier tipo de malestar, pelea o situación de violencia sea tipificado como buling.

En una de las dos instituciones de la localidad de Avellaneda (escuela 1) donde realizamos el trabajo de campo, nos encontramos con la particularidad de que existía un grupo que se autodenominaba "Los Bullying". De hecho, una vez enterados los estudiantes que estábamos en la institución haciendo entrevistas para una investigación, uno de ellos interpeló a uno de nosotros diciendo: "vos me tenés que entrevistar a mí porque yo soy Bullying". Frente a esa situación, además de realizar las respectivas entrevistas individuales, decidimos hacer un grupo focal con los estudiantes que integraban ese grupo (Grupo Focal 5to. Año, Escuela 1 - Avellaneda). A partir de este caso, pudimos advertir ciertos efectos simbólico-subjetivos que tienen en el ámbito escolar los discursos hegemónicos de sentido común que se construyen y circulan en los medios de comunicación.

\section{El caso de "Los Bullying"}

No es casualidad que un estudiante se haya presentado como "bullying" y que sea parte de un grupo que se autodenominaba "Los Bullying". Dicho nombre surgió cuando la exdirectora de la 
escuela, en una de las repetidas llamadas de atención en preceptoría, le dijo a uno de los alumnos que "tenía principio de bullying". Él mismo nos relató cómo fue:

L: La directora agarró y me dijo que me tenía que cambiar a la tarde porque tenía miedo porque yo tenía principio de Bullying. [...] Y yo eso lo traté de tomar como más tranqui; como diciendo "ya está, ésta piensa esto", yo sé que no lo soy. Me lo traté de tomar como más divertido. No es divertido lo que me dijeron, ja, pero igual lo tomé como más liviano y lo tomé más a la forma de chiste. Y ahí empezamos a decir a lo que hacemos nosotros, a decirnos "Bullying". A mí me empezaron a decir Bullying... me decían "eh, Bullying, Bullying, Bullying". Y después ya empezamos todo el grupo a decirnos "Bullying".

P: No sólo vos sino el grupo...

L: Ahora somos "Los Bullying" [se ríe].

(Luciano, 5to. Año, Escuela 1 - Avellaneda).

Cuando la exdirectora le imputa a Luciano un "principio de bullying", le adjudica un atributo desacreditador (estigma) basado en rasgos personales (Goffman, 2006). Lo "diagnostica", en términos médicos, como un joven con problemas de corte psicopatológico, justificando de esa forma su modo de comportarse, sin enmarcarlo en el contexto social más amplio de interrelaciones en el cual se produce. Así, la responsabilidad recae sobre el estudiante quedando exenta de reflexión la dinámica institucional de la escuela y el rol de los docentes y las autoridades. A pesar de que Luciano asumió con gracia la etiqueta que le adjudicó la autoridad de la escuela, incorporó la noción de buling como una enfermedad.

Si bien los estudiantes de este grupo toman de forma jocosa el término e intentan hacer de éste una insignia grupal, también muestran cierta preocupación respecto de ser encasillados dentro de la escuela como lo que ellos consideran "buling en de verdad"

P: Volvamos un poco al tema del bullying... Ustedes saben lo que significa?

Varios: Sí.

$\mathrm{H}$ : Una persona que se abusa de los demás y no le importa la otra persona; bardea sin que quiera herir a la gente.

$\mathrm{N}$ : Agresión constante hacia otro produciendo daños psicológicos o otras consecuencias. Es eso, el daño a los otros. Como quemarle la cabeza a alguien.

G: No solamente puede ser físico sino también verbal. A nosotros nos dicen que es más verbal, nosotros no le pegamos a nadie. Eso es lo que nos dicen.

$P: O k$, ¿a ustedes les dicen que hacen esto que me están contando?

$\mathrm{S}$ : A él le dijeron eso [señalando con la cabeza a Luciano].

G: No, y el otro día también nos lo dijeron.

$\mathrm{N}$ : Porque le tiraron un bollo de papel en la cabeza a la profesora.

S: Le tiraron, bueeee...

P: ¿Quién le tiró?

L y H: Él [señalando a Sergio]

$\mathrm{S}$ : No, pero después ellos me amenazaron. 
$\mathrm{H}$ : Porque nosotros le dijimos... la gracia era tirarle un bollito de papel así [lo muestra con las manos haciendo referencia a algo pequeño]. Hice un bollo y la gracia era tirarle un papel [emula su lanzamiento]. No, él le hizo PUM! [hace un gesto con los brazos de lanzamiento fuerte y de que el papel le dio en la cabeza a la profesora. Todos se ríen]. Le dio vuelta la cabeza, la mato. Le dejó un chichón así.

$\mathrm{N}$ : siempre que uno hace una cosa así le decimos que no lo haga porque después nos tildan que somos bullying de verdad porque... [hablan al mismo tiempo, no se escucha].

(Grupo Focal 5to. Año, Escuela 1 - Avellaneda).

Tanto el etiquetamiento impuesto por parte de la exdirectora como las sanciones punitivas que recibían, tales como apercibimientos o la prohibición de salir al recreo, reducen la problemática a una cuestión dicotómica de víctima/victimario, inocentes/culpables, sanos/insanos que no permite abordarla en su complejidad, sino más bien contribuye a reforzar y fijar los "perfiles" y roles de los estudiantes.

En este marco, resulta enriquecedor recuperar los aportes de Bourdieu $(2012,2014)$ sobre el estudio de las taxonomías escolares y sociales, las cuales, en cuanto actos simbólicos de clasificación, son instrumentos de conocimiento práctico que contribuyen a estructurar nuestra percepción sobre el mundo social y el sentido de nuestras acciones. Las nominaciones hacia los jóvenes son actos productivos que tienen efecto de verdad sobre sus experiencias de subjetivación. Ellas los atraviesan en la construcción de su autoestima e identidad pudiendo incidir en su trayectoria escolar y social (Kaplan, 2008a).

La imposición de un nombre implica asignarle a alguien una esencia social por la cual se institucionaliza una identidad. De este modo, "el acto de institución es un acto de comunicación, pero de un tipo específico: notifica a alguien su identidad, en el doble sentido de que se la expresa y se la impone al expresarla ante todos, [...] notificándole de este modo con autoridad lo que es y lo que tiene que ser (Bourdieu, 2014: 103).

Se trata de un poder que al enunciar también contribuye performativamente a construir el mundo nombrado.

\section{Conclusiones}

A lo largo de este artículo se han caracterizado e interpretado las prácticas discursivas producidas en trabajos académicos, en la prensa escrita y en la comunidad educativa sobre la problemática del buling. Desde el análisis socioeducativo, se identifica una convergencia discursiva que despliega una mirada individualizante para explicar el fenómeno.

Hemos observado cómo en los medios de comunicación se divulgan de forma simplificada resultados de investigación articulándolos con máximas de sentido común. Esto propicia que categorías del campo científico que adquieren dominio público y popularidad pasen a formar parte del sustrato de conocimientos comunes, descontextualizando o distorsionándose su sen- 
tido original. Cabe recordar, como lo hacía Bourdieu (2014), que el discurso científico, dado su reconocimiento social, tiene un efecto simbólico que tiende a consagrar aquello que señala. La utilización de términos y conceptos provenientes del campo académico constituye un recurso más, un argumento, en las luchas por las clasificaciones sociales para imponer una determinada visión del mundo social.

Justamente, un hallazgo significativo de este trabajo consistió en la identificación de una inflación semántica del término buling como operación discursiva en las coberturas periodísticas y en los discursos que circulan en las escuelas. A partir de una diversificación y abuso del vocablo, cualquier tipo de conflicto, malestar o situación de violencia puede ser tipificada de ese modo.

A partir de una impronta psicologicista, los discursos sobre buling focalizan su explicación sobre la dimensión conductual y psíquica de los agresores y de las víctimas, interpretando sus comportamientos como patologías individuales. De allí que, como vimos en los fragmentos de la prensa escrita, varios de los profesionales que son consultados planteen la posibilidad de detectar o pronosticar quiénes tienen tendencias a ser "matones o víctimas" según los trastornos que sufran. Particularmente, consideramos que la construcción de "perfiles" típicos, que devienen en estereotipos, provee explicaciones de orden individual sobre los modos de comportarse de los estudiantes, quedando exento de reflexión el contexto en el que se producen las relaciones intersubjetivas y el sentido que los jóvenes le otorgan a sus acciones. Asimismo, al centrar las causas del problema en atributos personales, las responsabilidades recaen sobre la víctima, dada su "escasez de habilidades sociales", y sobre el agresor, por su "prepotencia dominadora".

Este tipo de perspectiva alimenta una mirada criminalizante cuando ciertos estudios ( $\mathrm{Fa}$ rrington et al., 2012; Olweus, 1996) sostienen que es posible predecir que los jóvenes agresivos que se comportan como hostigadores corren un riesgo claramente superior de caer en otras conductas reprobables tales como la delincuencia y el abuso de psicofármacos. Vincular ciertos comportamientos de los estudiantes con "probables" actos de delincuencias incita a criminalizar a los jóvenes (Kaplan, 2014). Asimismo, la impronta criminológica del discurso del buling también puede observarse en la legislación que existe en algunos países donde se equiparan situaciones de acoso escolar con infracciones tipificadas penalmente, promoviendo resoluciones judiciales más que pedagógicas (Campelo, 2016).

Los discursos patologizantes esencializan el comportamiento social de los jóvenes e individualizan las responsabilidades, anticipando un estigma social que recae sobre los alumnos tipificados como "hostigadores" u "hostigados", sin poder luego desprenderse fácilmente de esa caracterización. Esto pudimos ejemplificarlo a partir del grupo de estudiantes que se autodenominó como "Los bullying" a partir de la etiqueta impuesta por la directora a uno de ellos. Se parte del supuesto de que la violencia es una propiedad dada y una forma de relacionarse intrínseca de determinados individuos o grupos. Sin embargo, en coincidencia con los hallazgos de otros 
investigadores (Kaplan, 2013, Paulín, 2015), consideramos que la violencia en las escuelas está asociada a determinadas condiciones de producción materiales, simbólicas e institucionales. Es decir, que se emplaza en un macrocosmos social.

Si bien este enfoque considera las relaciones de poder asimétricas entre los estudiantes, aíslan los conflictos reduciéndolos a una lógica entre víctimas, victimarios, espectadores y seguidores. Diversas investigaciones (di Napoli, 2020; Mutchinick, 2018, Paulín, 2015, Kaplan, 2017) aducen que este enfoque deja escapar diferentes formas de microviolencia que no se centran en casos o estudiantes específicos, pero que emergen como producto de la interacción entre los diferentes grupos de pares.

El presente trabajo es de relevancia en tanto es uno de los primeros estudios latinoamericanos que articulan los discursos mediáticos, las percepciones de actores de la comunidad educativa y el discurso académico sobre el fenómeno del buling. Es importante destacar que los análisis presentados posibilitan pensar los problemas y desafíos de la visibilización de la escuela y sus actores en los distintos ámbitos discursivos y sus efectos de sentido en la vida en las escuelas.

\section{Referencias}

Bourdieu, P. (2014). La fuerza de la representación. En Bourdieu, P. (E. Martínez, trad.). ¿Qué significa hablar? Economía de los intercambios lingüísticos. Buenos Aires: Akal, 111-121.

Bourdieu, P. (2012). La distinción: criterio y bases sociales del gusto. Buenos Aires: Taurus.

Bourdieu, P. (2006). Intelectuales, política y poder. Buenos Aires: Eudeba.

Campelo, A. (2016). Bullying y criminalización de la infancia. Cómo intervenir desde un enfoque de derechos. Buenos Aires: Noveduc.

Castillo, C.; M. Pacheco (2008). Perfil del maltrato (bullying) entre estudiantes de secundaria en la ciudad de Mérida, Yucatán. Revista Mexicana de Investigación Educativa, 13(38), 825-842.

Castro, A. (2013). Bullying blando, bullying duro y ciberbullying. Rosario: Homo Sapiens.

Cerezo, F. (2009). Bullying: análisis de la situación en las aulas españolas. International Journal of Psychology \& Psychological Therapy, 9(3), 367-378.

Defensor del Pueblo (2007). Violencia escolar: el maltrato entre iguales en la Educación Secundaria Obligatoria. Madrid.

Del Rey, R.; R. Ortega (2007). Violencia escolar: claves para comprenderla y afrontarla. Escuela Abierta, (10), 77-89.

Di Napoli, P. (2020). Sociodinámica de los conflictos juveniles en el espacio escolar. Aletheia, 12(1), 15-40. https://virtual.cinde.org.co/index.php/ALETHEIA/article/view/519 
Di Napoli, P. (2018). Reflexiones críticas sobre la noción de bullying desde un caso de estudio. Un análisis de las luchas simbólicas por el poder de nominación en el ámbito escolar. Espacios en blanco. Serie indagaciones, 28(2), 131-159.

Elias, N. (1999). Sociología fundamental. Barcelona: Gedisa.

Farrington, D.; F. Lösel; M. Ttofi; N. Theodorakis (2012). School Bullying, Depression and Offending Behavior later in Life: An Updated Systematic Review of Longitudinal Studies. Estocolmo: National Council for Crime Prevention.

Fereday, J.; E. Muir-Cochrane (2006). Demonstrating Rigor Using Thematic Analysis: A Hybrid Approach of Inductive and Deductive Coding and Theme Development. International Journal of Qualitative Methods, 5(1), 80-92.

Goffman, E. (2006). Estigma. La identidad deteriorada. Buenos Aires: Amorrortu.

Herrera-López, M.; E. Romera; R. Ortega-Ruiz (2018). Bullying y cyberbullying en Latinoamérica. Revista Mexicana de Investigación Educativa, XXIII(76), 125-155.

Kaplan, C. (2017). La vida en las escuelas. Esperanzas y desencantos de la convivencia escolar. Rosario: Homo Sapiens Ediciones.

Kaplan, C. (2014). La judicialización de la vida educativa. El bullying como categoría de naturaleza política. II Jornadas Internacionales "Sociedades Contemporáneas, Subjetividad y Educación". Buenos Aires. http://iice.institutos.filo.uba.ar/sites/iice.institutos.filo.uba.ar/files/ Kaplan,\%20Carina.pdf

Kaplan, C. (2013). Culturas estudiantiles: sociología de los vínculos en la escuela. Buenos Aires: Miño y Dávila.

Kaplan, C. (2008a). Talentos, dones e inteligencias. El fracaso escolar no es un destino. Buenos Aires: Colihue.

Kaplan, C. (2008b). Comportamiento individual y estructura social: cambios y relaciones. Una lectura desde Norbert Elias. En Kaplan, C. (coord.). La civilización en cuestión. Escritos inspirados en la obra de Norbert Elias. Buenos Aires: Miño y Dávila, 151-168.

Martín, E. (2014). Mentiras, inconsistencias y ambivalencias. Teoría de la acción y análisis de discurso. Revista Internacional de Sociología, 72(1), 115-138.

Menesini, E. (2009). El acoso en la escuela: desarrollos recientes. En R. Ortega, F. Mazzone, Educación en contextos de violencia y violencia en contextos educativos. Reflexiones y experiencias desde las acciones de intercambio en Italia. Roma: EUROsocial-CISP, 3-28.

Mutchinick, A. (2018). Modos de abordar las humillaciones entre estudiantes. Un estudio desde la perspectiva de alumnos de educación secundaria. Aposta. Revista de Ciencias Sociales, 77, 101-131.

Olweus, D. (1996). Problemas de hostigamiento y de víctimas en la escuela. Perspectivas, XXVI(2), 357-389.

Olweus, D. (1998). Conductas de acoso y amenaza entre escolares. Madrid: Morata. 
Ortega, R. (2006). La convivencia: un modelo de prevención de la violencia. En Moreno, A.; M. González; P. Soler; P. Álvarez. La convivencia en las aulas: problemas y soluciones. Madrid: Ministerio de Educación y Ciencia-Secretaría General Técnica-Subdirección General de Información y Publicaciones, 29-48.

Ortega, R.; J. Mora-Merchán (1997). Agresividad y violencia. El problema de la victimización entre escolares. Revista de Educación, (313), 7-27.

Paulín, H. (2015). Hacia un enfoque psicosocial crítico de la violencia escolar. Aportes desde un estudio con estudiantes de la ciudad de Córdoba, Argentina. Universitas Psychologica, 14(5), 1751-1762.

Resett, S. (2011). Aplicación del Cuestionario de Agresores/víctimas de Olweus a una muestra de adolescentes argentinos. Revista de Psicología de la Universidad Católica Argentina, 13(7), 27-44.

Román, M.; F. Murillo (2011). América Latina: violencia entre estudiantes y desempeño escolar. Revista CEPAL, (104), 37-54.

Saez, V. (2019). La criminalización mediática en los discursos sobre la escuela argentina. Psicoperspectivas, 18(1), 144-156. https://doi.org/10.5027/psicoperspectivas-vol18-issue1-fulltext-1326

Saez, V. (2020). Luchas simbólicas en la visibilización de la escuela. Un análisis de la construcción de los victimarios de las situaciones de violencia en los medios de comunicación. Revista Colombiana de Educación, 1(78). https://doi.org/10.17227/rce.num80-6974

Smith, P.; S. Sharp (1994). School Bullying. Insights and Perspectives. Nueva York: Routledge.

Trianes Torres, M. V. (2000). La violencia en contextos escolares. Archidona: Aljibe.

Zequinão, M.; P. Medeiros; B. Pereira; F. Cardoso (2016). Bullying escolar: um fenômeno multifacetado. Educação e Pesquisa, 42(1), 181-198. 